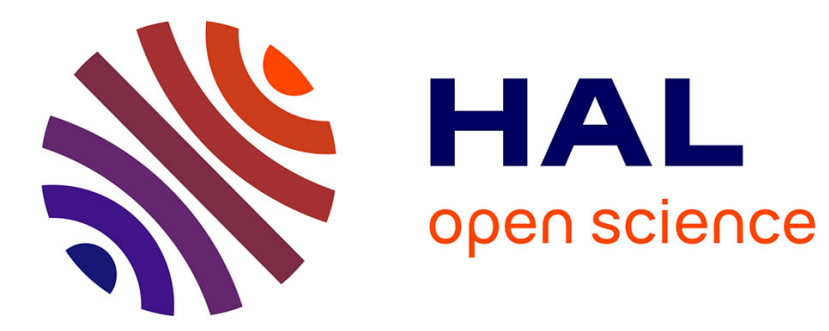

\title{
From alpha to beta functional and phylogenetic redundancy
}

\author{
Carlo Ricotta, Fabien Laroche, László Szeidl, Sandrine Pavoine
}

\section{To cite this version:}

Carlo Ricotta, Fabien Laroche, László Szeidl, Sandrine Pavoine. From alpha to beta functional and phylogenetic redundancy. Methods in Ecology and Evolution, 2020, 11 (4), pp.487-493. 10.1111/2041210X.13353 . hal-02917071

\section{HAL Id: hal-02917071 \\ https://hal.science/hal-02917071}

Submitted on 18 Aug 2020

HAL is a multi-disciplinary open access archive for the deposit and dissemination of scientific research documents, whether they are published or not. The documents may come from teaching and research institutions in France or abroad, or from public or private research centers.
L'archive ouverte pluridisciplinaire HAL, est destinée au dépôt et à la diffusion de documents scientifiques de niveau recherche, publiés ou non, émanant des établissements d'enseignement et de recherche français ou étrangers, des laboratoires publics ou privés. 
From alpha to beta functional and phylogenetic redundancy

\section{Carlo Ricotta $^{1, \star}$ Fabien Laroche ${ }^{2}$, László Szeidl ${ }^{3}$ and Sandrine Pavoine ${ }^{4}$}

${ }^{1}$ Department of Environmental Biology, University of Rome 'La Sapienza', Rome, Italy.

${ }^{2}$ Irstea, UR EFNO, F-45290 Nogent-sur-Vernisson, France.

${ }^{3}$ John von Neumann Faculty of Informatics, Óbuda University, 1034 Budapest, Hungary.

${ }^{4}$ Centre d'Ecologie et des Sciences de la Conservation (CESCO), Muséum National d'Histoire Naturelle, CNRS, Sorbonne Université, 75005 Paris, France.

*Corresponding author. E-mail: carlo.ricotta@uniroma1.it

\section{Abstract}

1. Plot-level redundancy or alpha redundancy is usually defined as the fraction of species diversity not expressed by functional or phylogenetic diversity. Redundancy is zero when all species in one plot are maximally dissimilar from each other. By contrast, redundancy tends to its maximum if the functional or phylogenetic differences between species tend to be minimal.

2. To explore the ecological drivers of community assembly, ecologists also use dissimilarity measures between pairs of plots (a component of beta diversity). Traditional dissimilarity measures summarize compositional differences between pairs of plots based either on species presence and absence data or on species abundances, thus attributing equal distinctiveness between any two species.

3. In the last decades a number of dissimilarity measures which incorporate information on functional or phylogenetic differences among species have been proposed. Based on such improved measures, we can define an index of beta redundancy for a pair of plots as the fraction of species dissimilarity not expressed by functional or phylogenetic dissimilarity.

4. A necessary condition to get a meaningful index of beta redundancy is that for a given pair of plots, the functional or phylogenetic dissimilarity is always lower or equal to the corresponding species dissimilarity. However, many of the existing indices of functional or phylogenetic dissimilarity can lead to values greater than for species dissimilarity.

5. The aim of this paper is thus to introduce a new family of tree-based measures of phylogenetic and functional dissimilarity that conform to this requirement. To show the behavior of the proposed measures, a worked example with data on Alpine vegetation is used.

Keywords: Bray-Curtis index, functional and phylogenetic dissimilarity, functional dendrogram, hierarchical clustering, phylogenetic tree, species-based dissimilarity. 


\section{Introduction}

The maintenance of ecosystem processes under ongoing perturbations causing local species extinctions requires the presence of functionally similar species. This property is usually known as functional redundancy (Walker, 1992; Naeem, 1998). When several species perform similar functions but differ in their responses to disturbances, functional redundancy may enhance community stability, while providing insurance against the loss of ecosystem functions due to a disturbance (Yachi \& Loreau, 1999). Therefore, if functional redundancy is high, it is more uniqueness within plots (the complement of redundancy) as the ratio of two diversity measures: the Rao quadratic entropy $Q$, which is calculated using the species abundances and their trait dissimilarities and the Simpson index $S$, which considers all species maximally dissimilar from each other such that $U_{\alpha}=Q / S$. Functional uniqueness thus measures the diversity decrease that is obtained by including trait dissimilarities in the calculation of plot-level diversity or alpha diversity. Similarly, since $Q \leq S$, alpha-redundancy can be interpreted as the fraction of species diversity not expressed by functional diversity: $R_{\alpha}=1-U_{\alpha}=(S-Q) / S$ with $R_{\alpha}$ ranging between zero and one (Pillar et al., 2013). Redundancy is zero when all species in the assemblage are maximally dissimilar from each other. In this case, Rao's quadratic diversity is equal to the Simpson index. By contrast, if all species in the assemblage are functionally identical, we have $Q=0$ and hence $R_{\alpha}=1$.

In addition, community ecologists increasingly recognize that ecological processes may also be affected by evolutionary differences among species. This is because the branching structure of 61 phylogenetic trees generally mirrors the accumulation of phenotypic differences among species, which may reflect the complexity of ecosystem functioning better than a reduced set of trait 
differences (Tucker et al., 2017). As for functional redundancy, we can thus define phylogenetic redundancy as the diversity decrease that is obtained by considering the evolutionary relationships among species in the calculation of diversity. Ricotta et al. (2018) thus proposed a normalized measure of plot-level phylogenetic redundancy or alpha redundancy in the range [0, 1] that relates the observed phylogenetic diversity of a given species assemblage to the value of a hypothetical assemblage in which all species originate directly from the same ancestor node giving rise to a star phylogeny. For details, see Ricotta et al. (2018).

Apart from plot-level indicators, ecologists also use dissimilarity measures between pairs of plots to explore the complex mechanisms that drive ecosystem functioning. Classical dissimilarity measures usually summarize compositional differences between pairs of plots based either on species incidence or abundance data, thus attributing equal distinctiveness between any two species (for review, see Podani, 2000 or Legendre \& Legendre, 2012). More recently, a number of functional/phylogenetic dissimilarity measures, which incorporate information on functional or phylogenetic differences among species have been proposed (Rao, 1982; Clarke \& Warwick, 1998; Izsák \& Price, 2001; Pavoine, Dufour, \& Chessel, 2004; Chiu, Jost, \& Chao, 2014; Pavoine \& Ricotta, 2014; Pavoine, 2016; Chao et al., 2019). Such new dissimilarity measures are expected to correlate more strongly with ecosystem processes, as species directly or indirectly influence these processes via their traits (Mason \& de Bello, 2013).

$$
\text { Plot-to-plot dissimilarity is closely related to the concept of beta diversity proposed by }
$$
Whittaker (1960) to define the amount of species turnover in a given set of plots. Indeed, evaluation of beta diversity is commonly based on the average dissimilarity between pairs of plots (Koleff, Gaston, \& Lennon, 2003; Legendre \& De Cáceres, 2013, but see Baselga, 2013). Therefore, we can define a measure of beta redundancy $R_{\beta}$ for a pair of plots as the fraction of species dissimilarity not expressed by functional or phylogenetic dissimilarity. According to this definition, a necessary condition to obtain a normalized index of beta redundancy in the range [0, 
88 1] is that for a given pair of plots, the functional (or phylogenetic) dissimilarity $D_{F}$ (or $D_{P}$ ) is 89 always lower or equal to their species dissimilarity $D_{S}$, where $D_{S}$ is the compositional 90 dissimilarity of a hypothetical pair of plots with the same species abundances of the actual pair

91 of plots in which all species are considered equally and maximally dissimilar from each other. In 92 this case, beta redundancy can be summarized as:

93

$R_{\beta}=1-U_{\beta}=\left(D_{S}-D_{F}\right) / D_{S}$

However, under particular circumstances, many of the existing indices of functional or phylogenetic dissimilarity can lead to values greater than for species dissimilarity, thus violating the basic requirement that $D_{F}, D_{P} \leq D_{T}$ (see Appendix $\mathrm{S} 1$ ). The aim of this paper is to introduce a new family of tree-based measures of phylogenetic and functional dissimilarity that conform to the requirement $D_{F}, D_{P} \leq D_{T}$. A worked example with published data on Alpine plant communities along a primary succession on a glacier foreland in northern Italy is used to illustrate our approach.

\section{New tree-based measures of phylogenetic beta redundancy}

Phylogenetic trees have long been used to represent the evolutionary history and relationships among groups of organisms, such as species (Podani 2017). In ecology, phylogenetic trees have been widely adopted to analyze the phylogenetic component of biodiversity (Tucker et al. 2017). Likewise, most measures of phylogenetic dissimilarity take into account the branching pattern of the species phylogeny (Chiu, Jost, \& Chao, 2014; Pavoine, 2016). For a given assemblage, the terminal nodes of the phylogenetic tree usually represent the species in the assemblage, while the

111 interior nodes are 'hypothetical taxonomic units' (i.e., the hypothetically most recent common 112 ancestor of all descendent taxa in the phylogenetic tree; Farris, 1970). If the branch lengths are 
113 proportional to the time of divergence among species, all species are at the same distance from 114 the root node and the tree is called 'ultrametric'. A general method for incorporating the 115 branching pattern of ultrametric trees like that in Figure 1 in the calculation of a wide range of 116 phylogenetic diversity metrics has been developed by Pavoine, Love, \& Bonsall (2009) and 117 Chao, Chiu, \& Jost (2010).

118 For an ultrametric phylogeny composed of $N$ species, let $A$ and $B$ be two plots and $x_{A i}$ be the 119 abundance of species $i(i=1,2, \ldots, N)$ in plot $A$. The phylogenetic tree is first divided into $K$ 120 evolutionary periods as follows: the tree is sliced at each node and the node ages are labeled in 121 temporal order as $t_{k}$ with $t_{0}=T$ (the age of the root node) and $t_{K}=0$ (the present-day time). The root node can either be the common ancestor of the species in $A$ and $B$ or the common ancestor 123 of a larger group of species defined e.g. at regional scale, depending on the question at hand (see Ricotta et al., 2018).

Two adjacent slices delimit an evolutionary period $\Delta_{k}=t_{k-1}-t_{k}$ with $\sum_{k=1}^{K} \Delta_{k}=T$. Any given evolutionary period $k(k=0,1, \ldots, K)$ contains $M_{k}$ lineages $\left(M_{k} \leq N\right)$ with abundances $\chi_{k m}$ $\left(m=1,2, \ldots, M_{k}\right)$. The abundance of each lineage is obtained as the sum of the abundances of all its descendent species in the phylogenetic tree.

129 Given two plots $A$ and $B$, the corresponding lineage abundances $\chi_{A k m}$ and $\chi_{B k m}$ can be then 130 used for calculating a lineage dissimilarity $D_{k}$ between $A$ and $B$ for each node age $t_{k}$ of the tree. 131 The lineage dissimilarity at time $t_{K}$ is the usual present-day dissimilarity calculated from the 132 species abundances $x_{A i}$ and $x_{B i}$. The overall phylogenetic dissimilarity $D_{P}$ is then obtained by 133 averaging the measures $D_{k}$ over the time intervals of the corresponding evolutionary periods 134 (Ricotta et al., 2018): 
138 where $\tau_{k}=\Delta_{k} / T$ is the duration of the $k$-th evolutionary period after rescaling the tip-to-root

139 length to unit, and $\Delta_{0}$ (and hence $\tau_{0}$ ) $=0$ by definition. $D_{P}$ thus measures the phylogenetic 140 dissimilarity between plots $A$ and $B$ incorporating information about the branching pattern of the 141 species phylogeny and its lineage abundances.

142 If phylogenetic dissimilarity at time $t_{k}$ is calculated with the Bray-Curtis index (Bray \& 143 Curtis, 1957), the present-day species dissimilarity $D_{S}=D_{K}=\sum_{i=1}^{N}\left|x_{A i}-x_{B i}\right| / \sum_{i=1}^{N}\left(x_{A i}+x_{B i}\right)$ is 144 always greater than or equal to any other lineage dissimilarity $D_{k}$ (proof in Appendix S2). 145 Therefore, $D_{P} \leq D_{S}$ and we can get a normalized index of phylogenetic beta redundancy $\left(R_{P}\right)$ or beta uniqueness $\left(U_{P}\right)$ as $R_{P}=1-U_{P}=\left(D_{S}-D_{P}\right) / D_{S}$.

This measure represents the fraction of species dissimilarity between a pair of plots not expressed by phylogenetic dissimilarity. Note that for a pair of plots $A$ and $B$ with given species abundances $x_{A i}$ and $x_{B i}$, the uniqueness component $U_{P}=D_{P} / D_{K}$ can be interpreted as a measure of phylogenetic dissimilarity between both plots normalized by its maximum value. This maximum is the phylogenetic dissimilarity of a pair of plots with identical abundance vectors but in which all species had independent evolution. In phylogenetic terms, this means that all 153 terminal branches originate from a single polytomy at the root node, thus forming a star 154 phylogeny (Ricotta et al., 2018). Likewise, the complement of uniqueness, beta redundancy $R_{P}=1-U_{P}$, can be interpreted as a normalized measure of phylogenetic similarity in the range $[0,1]$.

\section{New tree-based measures of functional beta redundancy}

In principle, the same tree-based approach can be used to assess functional dissimilarity. This requires to organize functional differences between species to obtain a hierarchical structure as 
161 first suggested by Petchey \& Gaston (2002). Functional dendrograms can be constructed by first 162 calculating functional dissimilarities between species and then using any hierarchical clustering 163 approach to obtain a rooted tree from these dissimilarities with species as tips (Pavoine \& 164 Ricotta, 2019). There are several clustering methods for constructing a tree-like classification in 165 which species are aggregated into hierarchical clusters (Podani, 2000; Legendre \& Legendre, 166 2012). The structure of the resulting functional dendrogram is thus sensitive to the methods used 167 to define a tree hierarchy from functional dissimilarities between species.

168 Much of the discussion on tree-based functional measures has been focused around the selection of appropriate clustering methods (Podani \& Schmera, 2006; Petchey \& Gaston, 2007; Mouchet et al., 2008). Nonetheless, there is a lack of agreement over which method produces the 171 best representation of species distribution in hierarchical trait space. For example, Weitzman 172 (1992) developed an algorithmic measure of (functional) diversity, which can be geometrically 173 represented as the length of a rooted dendrogram, while Blackburn, Petchey, Cassey, \& Gaston 174 (2005) and Petchey \& Gaston (2007) suggested to adopt case by case the clustering algorithm 175 that maximizes the cophenetic correlation between pairwise species dissimilarities in trait space 176 and the corresponding pairwise dissimilarities across the functional dendrogram.

177 Podani \& Schmera $(2006,2007)$ argued that the ideal requirement of maximizing the 178 cophenetic correlation is hardly feasible in practice as the virtually infinite number of available 179 clustering procedures (see Lance \& Williams, 1966) would unnecessarily overcomplicate the 180 calculations. They suggested instead to use group average (i.e., unweighted pair group method 181 with arithmetic mean, UPGMA) as a standard clustering procedure. Mouchet et al. (2008) 182 proposed to use consensus trees, which incorporate features common to a number of different 183 clustering methods. Additional hierarchical clustering methods may rely on the maximization of 184 within-cluster homogeneity based on some a-priori defined criteria, such as variance, sum of 185 squares or within-cluster average similarity of objects (see Podani, 1989). 
In this paper, we used the UPGMA clustering method because it represents a good compromise between the methodological extremes of a continuous series of space-contracting and space-dilating algorithms (Gordon, 1999). Space-contracting algorithms, such as the single linkage method, tend to contract the input dissimilarity structure significantly. Therefore, they may leave some clusters undetected even if the input data space shows a clear group structure. Space-dilating algorithms, such as the complete linkage method, tend to produce a clustered structure even if it is not apparent in the input data space. Within this continuous series of clustering procedures, UPGMA generally preserves a large proportion of the input dissimilarity structure, thus tending to minimize the distortion induced by transforming the input dissimilarity matrix into a functional dendrogram (Podani \& Schmera, 2006).

As for the phylogenetic case, the functional dendrogram obtained from the UPGMA algorithm can then be used for calculating a measure of pairwise functional dissimilarity among plots $D_{F}$ together with the corresponding measures of functional redundancy and uniqueness $R_{F}=1-U_{F}=\left(D_{S}-D_{F}\right) / D_{S}$.

Note that, irrespective of the clustering method, the functional dendrogram needs to be rooted and scaled to unit height from the tips to the root, so that two species sharing no branch in the tree would have maximum possible functional differences between them (for details, see Appendix S3).

\section{Worked example}

\subsection{Data}

We analyzed the functional and phylogenetic beta redundancy of plant communities collected by Caccianiga et al. (2006) along a primary succession on glacial deposits. The data set is composed of 59 plots, each of about $25 \mathrm{~m}^{2}$ in size sampled above the tree line at the foreland of

211 site. Based on the age of the moraine ridges, the plots were assigned to three successional stages: 
early-succession (17 plots), mid-succession (32 plots) and late-succession (10 plots). For each

213 plot, species abundances were measured with a five-point ordinal scale transformed to ranks.

214 The 45 species sampled were classified in terms of Grime's (1974) plant strategy theory, as 215 competitors $(\mathrm{C})$, stress tolerators $(\mathrm{S})$ and ruderals $(\mathrm{R})$ with fuzzy-coded values in the range 0 216 100, such that the sum of $\mathrm{C}+\mathrm{S}+\mathrm{R}$ was equal to 100 (all data are available in Ricotta et al., 2016, 217 Appendix S2). A functional dissimilarity matrix between species was then obtained by applying 218 the Bray-Curtis coefficient to the CSR species classification. Finally, we used the phylogeny of 219 the 45 species available in Appendix A of Ricotta et al. (2015) after rescaling its tip-to-root 220 length to unit.

\subsection{Methods}

We calculated functional and phylogenetic beta uniqueness $U_{F}$ and $U_{P}$ for all pairs of plots in each successional stage. All calculations were done with a new R function "DP" available in Appendix S3. We next tested for differences in the dispersion of $U_{F}$ and $U_{P}$ among the three successional stages. Since the beta uniqueness of a pair of plots $A$ and $B$ is basically a normalized measure of dissimilarity between $A$ and $B$, we used the PERMDISP test (Permutational Analysis of Multivariate Dispersions) of Anderson (2006) to test for differences in the average dissimilarity of individual plots from their group centroid among successional stages. This test has been extensively used in ecology for comparing the beta diversity among groups of plots.

First, starting from the functional and phylogenetic beta uniqueness between pairs of plots, we calculated for each successional stage the normalized dissimilarity (uniqueness) of each individual plot from its group centroid. Next, a permutational t-test for pairwise differences in average dissimilarity from the group centroids is performed. P-values were obtained using 9999 pairwise permutations of the dissimilarities of individual plots from the corresponding group centroids. 


\subsection{Results}

The phylogenetic and functional dendrograms of the 45 species used in this study are shown in Appendix S4 and the behavior of functional and phylogenetic beta uniqueness along the successional stages is summarized in Table 1. As shown by Caccianiga (2006) and Ricotta et al. (2016, 2018) the colonization of the glacial deposits by the first pioneer species in the earlysuccessional stage is mainly driven by random dispersal mechanisms, while in the mid- and latesuccessional stages, a general tendency towards an increased structural homogeneity is observed. At the plot level, the increased uniformity of vegetation structure over time is associated with a functional and phylogenetic shift from early-successional ruderal forbs to late-successional stress-tolerator graminoids that goes together with an increase in functional and phylogenetic alpha redundancy.

Looking at the beta component of uniqueness, Table 1 shows that, due to the low structural homogeneity of the pioneer vegetation, the species, functional and phylogenetic turnover among the early-successional plots is generally higher than that of the mid-and late successional plots. More specifically, while traditional species turnover does not differ significantly among the three successional stages, the functional turnover and uniqueness of the 17 plots in the early successional stage are both significantly higher than those in the mid- and late successional stages. This means that in the mid- and late-successional stages the species in one plot tend to be replaced by functionally related species in the other plot. As a result, functional uniqueness $U_{F}$ tends to be low. By contrast, due to the more random nature of the dispersal mechanisms, in the early-successional stage, the species in one plot tend to be replaced by functionally unrelated (or, at least, less related) species. Therefore, functional uniqueness tends to be higher. The same 260 pattern is shown by the phylogenetic uniqueness, although in this case the statistical differences among the three successional stages are not always significant at $\mathrm{p}<0.05$. 


\section{Discussion}

In this paper, we introduced the notion of beta redundancy. In analogy to plot-level redundancy or alpha redundancy, beta redundancy can be defined as the fraction of species dissimilarity between pairs of plots not expressed by functional or phylogenetic dissimilarity. In its very essence, beta redundancy tells us to what degree the species-based similarity between two plots is associated to the functional or phylogenetic similarities among the species in both plots.

While functional and phylogenetic dissimilarity $D_{F}$ and $D_{P}$ are absolute measures that take into account the abundances and the functional or phylogenetic differences among the species in both plots, beta redundancy $R_{\beta}$ and its complement beta uniqueness $U_{\beta}=1-R_{\beta}$ are standardized coefficients that relate $D_{F}$ and $D_{P}$ to plot-to-plot species dissimilarity $D_{S}$. A fundamental requirement to get a meaningful index of beta redundancy is thus that $D_{F}, D_{P} \leq D_{S}$. However, contrary to common belief, many of the existing indices of functional or phylogenetic dissimilarity do not conform to this requirement. Therefore, they cannot be used to calculate an appropriate measure of beta redundancy. To solve this problem, we introduced a family of treebased indices of functional and phylogenetic dissimilarity that conform to this requirement. Regardless of whether beta redundancy is calculated from functional or phylogenetic data, $D_{F}$ and $D_{P}$ have a great potential for future research on community organization, as both measures are variations on the theme of the classical index of Bray \& Curtis (1957), the properties of which have been studied by ecologists for decades (e.g. Legendre \& De Cáceres, 2013; Ricotta \& Podani, 2017).

Being based on a hierarchical structure of species differences, the proposed phylogenetic measures take into account the branching pattern of the phylogenetic tree, which is the more natural way for describing the evolutionary relationships among the species. By contrast, a treebased representation of the species functional relationships may not be the most appropriate 
solution. However, if we accept the idea of Petchey and Gaston (2007, p. 1422) that from a

290 statistical viewpoint "the functional dendrogram can be thought of as a description of the 291 functional relationships shared by the species it includes, in the same way as a phylogenetic tree 292 describes phylogenetic relationships (although without any associated inference about 293 evolutionary relationships)", we obtain a powerful and flexible framework for the calculation of 294 different facets of functional diversity, richness and redundancy.

295 From a more technical viewpoint, our approach requires that the tip-to-root length $T$ of the phylogenetic or functional tree be normalized to the unit interval. This can be done either by apriori normalizing the (functional) dissimilarities among species in the range [0-1], or by aposteriori normalizing the length of the resulting dendrogram. Since there is no univocal way to normalize tree length, flexibility is important to adapt the way the data are transformed to the 300 specific objectives of our work. A short guide on how to normalize the length of a hierarchical dendrogram according to the specific question at hand can be found in Pavoine, Marcon, \& Ricotta (2016, Appendix S3).

In conclusion, we see this work more as a starting point for the summarization of beta redundancy rather than as an ultimate solution, and many questions remain still open. For example, how do the proposed beta redundancy metrics respond to large differences in species richness and evenness? (Here, it will also be important to explore which among rare and tree. For example, we could also consider evolutionary distances calculated from molecular data 312 before any tree is derived from these distances. Therefore, apart from tree-based indices, are 313 there other classes of measures of functional or phylogenetic dissimilarity which conform to the 314 requirement $D_{F}, D_{P} \leq D_{S}$ ? Which additional properties should these measures possess? In 
315 Appendix S1 we show that eight existing indices of plot-to-plot dissimilarity fulfill the 316 requirement $D_{F}, D_{P} \leq D_{S}$. However, for the same indices, we also show some unexpected and 317 counter-intuitive behaviors: part of these measures attribute positive dissimilarity values to two 318 identical plots, whereas, by definition, the dissimilarity between a plot and itself should be zero. 319 Other indices retain only nearest-neighbor distances between species for the calculation of 320 functional or phylogenetic dissimilarity (see Appendix S1 and Ricotta, Bacaro \& Pavoine, 2015). 321 Further studies should thus consider a range of properties fulfilled by any potential index of plot322 to-plot functional or phylogenetic dissimilarity, rather than focusing exclusively on the 323 requirement $D_{F}, D_{P} \leq D_{S}$.

324 Last but not least, in diversity theory alpha and beta diversity have been usually related to 325 gamma or regional diversity either by an additive model $\alpha+\beta=\gamma$ (McArthur, Recher, \& Cody, 326 1966) or by a multiplicative model $\alpha \times \beta=\gamma$ (Whittaker, 1960). Will it be possible to develop a 327 similar model for alpha and beta redundancy? These are critical questions, and their answers may 328 provide new tools for analyzing the complex processes that drive community assembly and 329 species co-occurrence.

\section{Acknowledgements}

332 We thank two anonymous reviewers for their comments. They both contributed to improve our 333 paper.

\section{Authors' Contributions}

336 C.R. and S.P conceived the ideas and formulated the research problem. F.L. and L.S. provided 337 important feedback which helped shape the research. C.R. and S.P. analyzed the data. C.R. took 338 the lead in writing the main text of the manuscript and S.P. in writing the appendixes. All authors 339 revised the manuscript critically and approved the final version.

\section{Data accessibility}

342 The data and the $\mathrm{R}$ code used in this paper are are deposited in the Dryad repository: 343 https://doi.org/10.5061/dryad.ttdz08ktg 


\section{References}

345 Anderson, M.J. (2006) Distance-based tests for homogeneity of multivariate dispersions. Biometrics, 62, 245-253.

Baselga, A. (2013) Multiple-site dissimilarity quantifies compositional heterogeneity among several sites, while average pairwise dissimilarity may be misleading. Ecography, 36, 124128.

Blackburn, T.M., Petchey, O.M., Cassey, P., \& Gaston, K.J. (2005) Functional diversity of mammalian predators and extinctions in island birds. Ecology, 86, 2916-2923.

Bray, J.R., \& Curtis, J.T. (1957) An ordination of the upland forest communities of southern Wisconsin. Ecological Monographs, 27, 325-349.

Caccianiga, M., Luzzaro, A., Pierce, S., Ceriani, R.M., \& Cerabolini, B.E.L. (2006) The functional basis of a primary succession resolved by CSR classification. Oikos, 112, 10-20.

Chao, A., Chiu, C.H., \& Jost, L. (2010) Phylogenetic diversity measures based on Hill numbers. Philosophical Transactions of the Royal Society B, 365, 3599-3609.

Chao, A., Chiu, C.-H., Villéger, S., Sun, I.-F., Thorn, S., Lin, Y.-C., Chiang, J.M., \& Sherwin, W.B. (2019) An attribute-diversity approach to functional diversity, functional beta diversity, and related (dis)similarity measures. Ecological Monographs, 89, e01343.

Chiu, C.H., Jost, L., \& Chao, A. (2014) Phylogenetic beta diversity, similarity, and differentiation measures based on Hill numbers. Ecological Monographs, 84, 21-44.

Clarke, K.R., \& Warwick, R.M. (1998). A taxonomic distinctness index and its statistical properties. Journal of Applied Ecology, 35, 523-531.

de Bello, F., Carmona, C.P., Lepš, J., Szava-Kovats, R., \& Pärtel, M. (2016) Functional diversity through the mean trait dissimilarity: resolving shortcomings with existing paradigms and algorithms. Oecologia, 180, 933-940.

Farris, J.S. (1970) Methods for computing Wagner trees. Systematic Zoology, 19, 83-92.

Gordon, A.D. (1999) Classification, Chapman \& Hall, London.

Grime, J.P. (1974) Vegetation classification by reference to strategies. Nature, 250, 26-31.

Izsák, C., \& Price, A.R.G. (2001) Measuring $\beta$-diversity using a taxonomic similarity index, and its relation to spatial scale. Marine Ecology Progress Series, 215, 69-77.

Koleff, P., Gaston, K.J., \& Lennon, J.J. (2003) Measuring beta diversity forpresence-absence data. Journal of Animal Ecology, 72, 367-382.

Lance, G.N., \& Williams, W.T. (1966) A generalized sorting strategy for computer classifications. Nature, 212, 218.

Legendre, P., \& De Cáceres, M. (2013) Beta diversity as the variance of community data: Dissimilarity coefficients and partitioning. Ecology Letters, 16, 951-963. 
Legendre, P., \& Legendre, L. (2012) Numerical Ecology. Elsevier, Amsterdam.

Mason, N.W.H., \& de Bello, F. (2013) Functional diversity: a tool for answering challenging ecological questions. Journal of Vegetation Science, 24, 777-780.

McArthur, R.H., Recher, H., \& Cody, M. (1966) On the relation between habitat selection and species diversity. American Naturalist, 100, 319-332.

Mouchet, M., Guilhaumon, F., Villéger, S., Mason, N.W.H., Tomasini, J.A., \& Mouillot, D. (2008) Towards a consensus for calculating dendrogram-based functional diversity indices. Oikos, 117, 794-800.

Naeem, S. (1998) Species redundancy and ecosystem reliability. Conservation Biology, 12, 3945.

Pavoine, S. (2016) A guide through a family of phylogenetic dissimilarity measures among sites. Oikos, 125, 1719-1732.

Pavoine, S., Dufour, A.B., \& Chessel, D. (2004) From dissimilarities among species to dissimilarities among communities: a double principal coordinate analysis. Journal of Theoretical Biology, 228, 523-537.

Pavoine, S., Love, M.S., \& Bonsall, M.B. (2009) Hierarchical partitioning of evolutionary and ecological patterns in the organization of phylogenetically-structured species assemblages: application to rockfish (genus: Sebastes) in the Southern California Bight. Ecology Letters, 12, 898-908.

Pavoine, S., Marcon, E., \& Ricotta, C. (2016) ‘Equivalent numbers' for species, phylogenetic or functional diversity in a nested hierarchy of multiple scales. Methods in Ecology and Evolution, 7, 1152-1163.

Pavoine, S., \& Ricotta, C. (2014) Functional and phylogenetic similarity among communities. Methods in Ecology and Evolution, 5, 666-675.

Pavoine, S., \& Ricotta, C. (2019) A simple translation from indices of species diversity to indices of phylogenetic diversity. Ecological Indicators, 101, 552-561.

Petchey, O.L., Evans, K.L., Fishburn, I.S., \& Gaston, K.J. (2007) Low functional diversity and no redundancy in British avian assemblages. Journal of Animal Ecology, 76, 977-985.

Petchey, O.L., \& Gaston, K.J. (2002) Functional diversity (FD), species richness and community composition. Ecology Letters, 5, 402-411.

Petchey, \& Gaston, K.J. (2007) Dendrograms and measuring functional diversity. Oikos, 116, $1422-1426$.

Pillar, V.P, Blanco, C.C., Müller, S.C., Sosinski, E.E., Joner, F., \& Duarte, L.D.S. (2013) Functional redundancy and stability in plant communities. Journal of Vegetation Science, 24, 963-974. 
Podani, J. (1989) New combinatorial clustering methods. Vegetatio, 81, 61-77.

Podani, J. (2000) Introduction to the Exploration of Multivariate Biological Data. Backhuys, Leiden, NL.

Podani, J. (2017) Different from Trees, more than Metaphors: Branching Silhouettes - Corals, Cacti, and the Oaks. Systematic Biology, 66, 737-753.

Podani, J., \& Schmera, D. (2006) On dendrogram-based measures of functional diversity. Oikos, $115,179-185$.

Podani, J., \& Schmera, D. (2007) How should a dendrogram-based measure of functional diversity function? A rejoinder to Petchey and Gaston Oikos, 116, 1427-1430.

Rao, R.C. (1982) Diversity and dissimilarity coefficients: a unified approach. Theoretical Population Biology, 21, 24-43.

Ricotta, C., Bacaro, G., Caccianiga, M., Cerabolini, B.E.L., \& Moretti, M. (2015) A classical measure of phylogenetic dissimilarity and its relationship with beta diversity. Basic and Applied Ecology, 16, 10-18.

Ricotta, C., Bacaro, G., Caccianiga, M., Cerabolini, B.E.L., \& Pavoine, S. (2018) A new method for quantifying the phylogenetic redundancy of biological communities. Oecologia, 186, 339346.

Ricotta, C., Bacaro, G., \& Pavoine, S. (2015) A cautionary note on some phylogenetic dissimilarity measures. Journal of Plant Ecology, 8, 12-16.

Ricotta, C., de Bello, F., Moretti, M., Caccianiga, M., Cerabolini, B.E.L., \& Pavoine, S. (2016) Measuring the functional redundancy of biological communities: a quantitative guide. Methods in Ecology and Evolution, 7, 1386-1395.

Ricotta, C., Laroche, F., Szeidl, L., \& Pavoine, S. (2020) Data from: From alpha to beta functional and phylogenetic redundancy. Methods in Ecology and Evolution doi:10.5061/dryad.ttdz08ktg

Ricotta, C., \& Podani, J. (2017) On some properties of the Bray-Curtis dissimilarity and their ecological meaning. Ecological Complexity, 31, 201-205.

Robuchon, M., Faith, D.P., Julliard, R., Leroy, B., Pellens, R., Robert, A., Thévenin, C., Véron, S., Pavoine, S. (2019) Species splitting increases estimates of evolutionary history at risk. Biological Conservation, 235, 27-35.

Tucker, C.M., Cadotte, M.W., Carvalho, S.B., Davies, T.J., Ferrier, S., Fritz, S.A., Grenyer, R., Helmus, M.R., Jin, L.S., Mooers, A.O., Pavoine, S., Purschke, O., Redding, D.W., Rosauer, D.F., Winter, M., \& Mazel, F. (2017) A guide to phylogenetic metrics for conservation, community ecology and macroecology. Biological Reviews, 92, 698-715.

Weitzman, M.L. (1992) On diversity. Quarterly Journal of Economics, 107, 363-405. 
449 Walker, B.H. (1992) Biodiversity and ecological redundancy. Conservation Biology, 6, 18-23.

450 Whittaker, R.H. (1960) Vegetation of the Siskiyou mountains, Oregon and California. 451 Ecological Monographs, 30, 279-338.

452 Yachi, S., \& Loreau, M. (1999) Biodiversity and ecosystem productivity in a fluctuating 453 environment: the insurance hypothesis. Proceedings of the National Academy of Sciences $454 \quad$ USA, 96, 1463-1468.

455

456 Supporting Information

457 Additional supporting information may be found online in the Supporting Information section at 458 the end of the article. 
459 Table 1. Mean (SD) dissimilarity values of each individual plot from its group centroid for the 460 three successional stages on the moraine deposits of the Rutor Glacier. Pairwise comparisons of 461 index differences between the successional stages were performed with permutational t-tests for 462 pairwise differences in average dissimilarity from the group centroids (9999 permutations). For 463 each dissimilarity coefficient, numbers followed by the same letter do not differ significantly at $\mathrm{P}$ $464<0.05 . D_{S}=$ species dissimilarity (Bray-Curtis dissimilarity); $D_{F}=$ functional dissimilarity; $D_{P}=$ 465 phylogenetic dissimilarity; $U_{F} / U_{P}=$ functional/phylogenetic uniqueness. 466 467 468

\begin{tabular}{cccc}
\hline & \multicolumn{3}{c}{ Functional data } \\
\hline & $\begin{array}{c}\text { Early-succ. } \\
\text { stage }\end{array}$ & $\begin{array}{c}\text { Mid-succ. } \\
\text { stage }\end{array}$ & $\begin{array}{c}\text { Late-succ. } \\
\text { stage }\end{array}$ \\
\hline$D_{S}$ & $0.399(0.071)^{\mathrm{a}}$ & $0.357(0.084)^{\mathrm{a}}$ & $0.354(0.101)^{\mathrm{a}}$ \\
$D_{F}$ & $0.118(0.034)^{\mathrm{a}}$ & $0.085(0.024)^{\mathrm{b}}$ & $0.073(0.033)^{\mathrm{b}}$ \\
$U_{F}=D_{F} / D_{S}$ & $0.203(0.045)^{\mathrm{a}}$ & $0.168(0.035)^{\mathrm{b}}$ & $0.144(0.69)^{\mathrm{b}}$ \\
\hline \multicolumn{4}{c}{ Phylogenetic data $^{\mathrm{a}}$} \\
\hline$D_{S}$ & $0.399(0.071)^{\mathrm{a}}$ & $0.357(0.084)^{\mathrm{a}}$ & $0.354(0.101)^{\mathrm{a}}$ \\
$D_{P}$ & $0.291(0.070)^{\mathrm{a}}$ & $0.214(0.053)^{\mathrm{b}}$ & $0.222(0.063)^{\mathrm{ab}}$ \\
$U_{P}=D_{P} / D_{S}$ & $0.498(0.076)^{\mathrm{a}}$ & $0.422(0.072)^{\mathrm{b}}$ & $0.427(0.101)^{\mathrm{ab}}$ \\
\hline
\end{tabular}


470 Figure 1. Ultrametric phylogenetic tree showing the evolutionary relationships among the species 471 of an artificial assemblage. The abundances of the five species are denoted as $x_{1}, \ldots, x_{5}$. By 472 slicing the phylogeny at each node we can define four non-overlapping evolutionary periods $\Delta_{k}$. 473 The age of each node is labeled as $t_{k}(k=0,1, \ldots, K)$ such that $t_{0}=T$ (the age of the root node), $474 t_{K}=0$ (the present-day time), and $\Delta_{k}=t_{k-1}-t_{k}$. Any evolutionary period $k$ contains $M_{k}$ lineages 475 with abundances $\chi_{k m}\left(m=1,2, \ldots, M_{k}\right)$. The abundances of each lineage along the phylogeny 476 are calculated by summing the abundances of all descendent species in the phylogeny. For 477 example, $\chi_{11}=\chi_{31}=x_{1}+x_{2}, \chi_{12}=x_{3}+x_{4}+x_{5}$, and $\chi_{K 1}=x_{1}$.

478

479

480

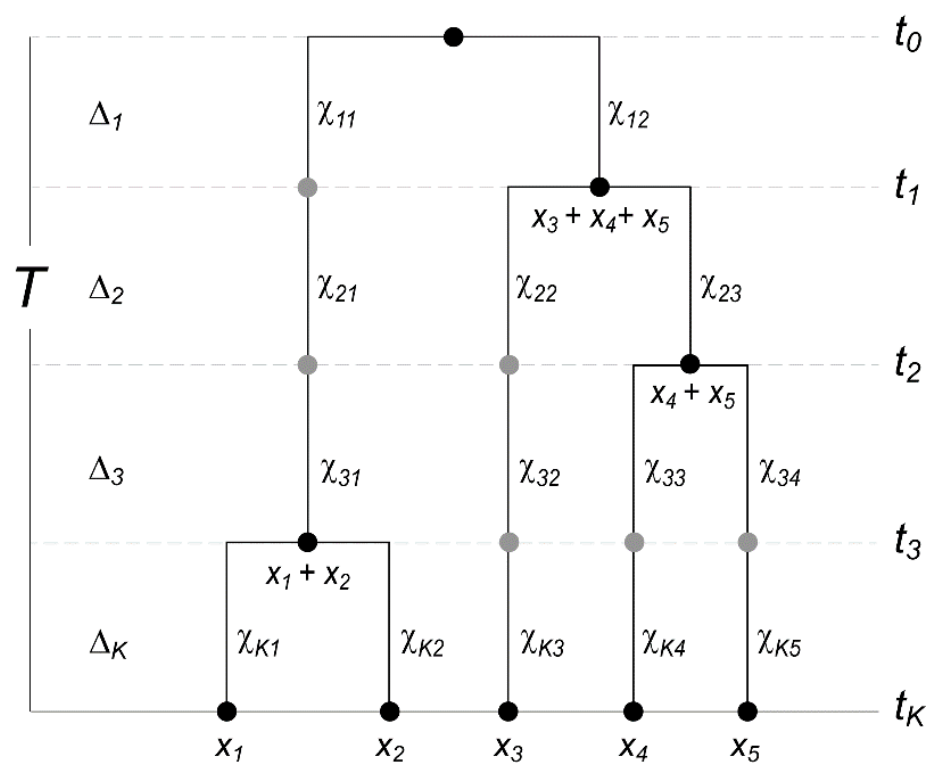

